EPiC Series in Computing
Volume 70, 2020, Pages 198-207
$\begin{gathered}\text { Proceedings of the 12th International Conference } \\ \text { on Bioinformatics and Computational Biology }\end{gathered}$

\title{
Exposure Measurements on Biomimetic Lobules Using Virtual Experiments to Help Improve IVIVE
}

\author{
Preethi Krishnan $^{1}$, Lopamudra Dutta ${ }^{1}$, Andrew K. Smith ${ }^{1}$, Glen E. P \\ Ropella $^{2}$, Ryan C. Kennedy ${ }^{1}$, and C. Anthony Hunt ${ }^{1}$ \\ ${ }^{1}$ Department of Bioengineering and Therapeutic Sciences, University of California, San \\ Francisco, CA, 94143 \\ ${ }^{2}$ Tempus Dictum, Inc., Milwaukie, OR 97222 \\ preethikrishnan27@gmail.com, lopamudra2008@yahoo.com, \\ drandrewksmith@gmail.com, gepretempusdictum.com, \\ ryan.c.kennedyealumni.nd.edu, a.hunteucsf.edu
}

\begin{abstract}
The in vitro-in vivo extrapolation (IVIVE) methods used currently to predict the hepatic clearance of new chemical entities are plagued by poorly understood inaccuracies. To begin identifying plausible sources, we challenge two of core hypotheses. Hypothesis-1: the intralobular micro-anatomical organization of hepatocytes (HPCs) can be abstracted away. By accepting that hypothesis, one can assume that intrinsic clearance per HPC is essentially the same in vitro and in vivo, and thus an IVIVE method can employ a simplified liver model, typically the "well-stirred" liver model. Hypothesis-2: when the simplified liver model is the "parallel tube model," drug concentration decreases exponentially from portal to central vein. When either simplified liver model is used, a core assumption is that intrinsic clearance is directly proportional to the unbound fraction of drug. A barrier to progress has been the fact that it is currently infeasible to challenge the two hypotheses using wet-lab experiments. In this work, we challenge virtual counterparts of the two hypotheses by experimenting on virtual mice in which hepatic disposition and clearance are consequences of concretized model mechanisms that have met several demanding requirements, including the following. The virtual liver's structure and organization are strongly analogous to those of an actual liver, and the hepatic disposition and clearance of several virtual compounds have achieved quantitative validation targets. We study two virtual compounds. Compound-1 simulates the extreme of low-clearance, highly permeable compounds. Compound-2 simulates a highly permeable compound exhibiting maximum intrinsic clearance. We simulate changes in unbound fraction by changing the probability (pEnter) that a Compound- 1 or -2 will enter an adjacent HPC during a simulation cycle. Compound-1 and -2 HPC
\end{abstract}


exposure rates do not decrease from portal to central vein: they increase, and that contradicts both hypotheses. Further, the relationship between exposure rates and pEnter is nonlinear. The insights achieved help explain the frequently reported underprediction of in vivo hepatic clearance values. We suggest that IVIVE methods can be improved by utilizing a liver model that couples a biomimetic representation of intralobular HPC organization with biomimetic representations of intrahepatic disposition dynamics.

\section{Introduction}

Predicting the hepatic clearance of new chemical entities is accomplished most frequently using in vitro-in vivo extrapolation (IVIVE) methods provide a comprehensive review [1]. The first stage is the determination of in vitro intrinsic clearance using isolated hepatocytes or microsomes. Next, a physiologically based model is used to scale and predict hepatic clearance in vivo. Most of those models represent hepatic clearance using either a well-stirred, or, less frequently, a parallel tube model of a liver. That IVIVE approach has failed frequently [2]. The sources of inaccuracies are still poorly understood [3-5]. Dissimilar biological and methodological factors are cited frequently as causes [6,7], and efforts are underway to identify and reduce IVIVE inaccuracies. For example, reported that, for low-clearance compounds, errors in determining the unbound fraction of drug are proportional to the errors in predicted clearance [8]. We are developing concretized model mechanism-based methods that are intended to be useful in disentangling and mitigating IVIVE inaccuracies incrementally $[9,10]$.

We focus on the veracity of two significant hypotheses (assumptions) — stated below—on which the above IVIVE methods are based. They have not been challenged directly using in vivo experiments because it is currently infeasible to do so. The alternative approach employed here is to experiment on virtual mice that meet several demanding requirements. To distinguish virtual mouse components, characteristics, and phenomena from real counterparts, we capitalize the former hereafter and, in several cases, append the prefix "v". For this work, the two most critical requirements are 1) the structure and organization of the vLiver within a vMouse is strongly analogous to that of an actual liver. The structural organization of vHPCs within vLobules maps directly and quantitatively to the measured 3D organization of HPCs within polyhedral hepatic lobules [9], and 2) during execution, the Disposition, Metabolism, and Clearance of vCompounds within vLivers, have achieved several quantitative validation targets [10-12]. Having met those requirements, we claim that the measurements of dynamic vCompound Disposition details of can be strongly analogous to in vivo counterparts. Thus, measures of Hepatic Disposition and Clearance details during Experiments can be used to challenge virtual counterparts of the two hypotheses and may provide insights that can be used to improve IVIVE methods.

The two hypotheses are related. Hypothesis- 1 focuses on use of the well-stirred liver model. It is that, absent drug-specific lobular-location influences, the intralobular micro-anatomical organization of HPCs within hepatic lobules can be extrapolated away. Consequently, in vitro and in vivo HPC exposure rates are essentially the same, within an acceptable degree of tolerance, and directly proportional to the unbound fraction of drug in extracellular fluid. It follows that the intrinsic clearance per HPC is also essentially the same in vitro and in vivo, and that greatly simplifies predicting in vivo clearance. Hypothesis- 2 focuses on use of the parallel tube liver model. It is that drug concentration within the liver decreases exponentially portal vein (PV)-to-central vein $(\mathrm{CV})$. At each PV-to-CV location represented within the model, equilibrium conditions exist, and down-stream exposure of HPCs to drug will be reduced proportionally due to upstream intracellular drug accumulation and clearance. Both the well-stirred and parallel-tube models assume that drug permeability is not a ratelimiting step in drug elimination. 
HPC exposure cannot be measured in vivo. Because we use discrete event, agent-oriented computational methods, we can measure the Exposure rates of idealized vCompounds-mobile chemomimetic objects - to vHPCs at different PV-to-CV locations following simulated oral dosing (see Results). We study two vCompounds. vCompound-1 maps to an idealized compound that does not undergo metabolism. Having zero Intrinsic Clearance, its Disposition is expected to map to that of highly permeable low-clearance compounds. vCompound-2 maps to an idealized compound that exhibits maximum intrinsic clearance in vitro. It can enter but does not exit a vHPC. Its Disposition and Clearance is expected to map to that of highly permeable high-clearance compounds.

A core assumption of IVIVE methods is that it is the unbound drug adjacent to HPCs that enters HPCs, thus becoming available to the intra-HPC processes responsible for drug metabolism and excretion. The unbound fraction of drug $\left(f_{u}\right)$ is an important variable in IVIVE predictions [13]. In the absence of active transport, researchers typically assume that the amount of compound within HPCs is directly proportional to the unbound fraction of drug in media or plasma [14]. We simulate changes in $\mathrm{f}_{\mathrm{u}}$ by changing the probability (pEnter) that a vCompound that is adjacent to a vHPCs will enter that vHPC within a simulation cycle. Thus, a change in $\mathrm{f}_{\mathrm{u}}$ from 1.0 to 0.3 maps directly to a change in pEnter from 1.0 to 0.3 .

Our results failed to support the two hypotheses. vHPC Exposure Rates for both vCompounds is dependent on PV-to-CV location, contrary to Hypothesis-2, and they do not decrease PV-to-CV: they increase. Pericentral (close to the CV) Exposure Rates for vCompound-1 are $>3.5$-fold larger than Periportal (close to the PV) Exposure Rates. Further, the relationship between Exposure Rates and pEnter is nonlinear. For vCompound-2, Exposure Rates and accumulation within vHPCs are relatively robust to changes in pEnter within the 0.3-1.0 range, independent of PV-to-CV location, whereas accumulation within Pericentral vHPCs is 3.2 -fold larger than that of Periportal vHPCs.

The biomimetic organization of vHPCs within the vLivers amplifies vCompound Exposure Rates of Pericentral vHPCs relative to upstream Periportal vHPCs, which helps explain the nonlinear consequences of changing pEnter. These insights achieved may help explain the frequently reported underprediction of in vivo hepatic clearance values when using established IVIVE methods. We suggest that IVIVE methods can be improved by utilizing a liver model, such as the vLiver, that couples a biomimetic representation of the structural organization of HPCs with biomimetic representations of Intrahepatic vCompound dynamics.

\section{Methods}

\subsection{Simulation Details}

Experiments were conducted using vLivers within vMice that was created using the Java-based MASON multiagent toolkit. Using agent-oriented techniques allows the vLiver to be biomimetic in multiple ways, such as having Cell objects respond to external and internal stimuli. Also, the concrete nature of a vLiver allows its features to be measured and annotated. The vLiver is biomimetic in part because mappings can be established between virtual and biological features and measures. vLiver components can be reused for different experiment use cases and versioning allows one component to evolve somewhat independent of others. Experiments are not intended for predicting the pharmacokinetic properties of particular compounds. Rather, they are used to challenge model mechanism-based hypotheses and to explore the behaviour spaces of model mechanism variants. Experiments are executed using Google Compute Engine running Linux. The R programming language is used for data analyses. The entire toolchain, including the operating system, configurations, and I/O handling is open-source. The data presented herein, along with the code, are available from project websites (https://simtk.org/projects/aili and https://simtk.org/home/isl/), and are available to be licensed as open data. 


\subsection{Virtual Liver and Virtual Compounds}

Because the vLiver is a concretized analogy of an actual liver, features do not map 1:1 to biological counterparts. vLobules map to a small random sample of possible lobular flow paths within a whole liver and the total volume of associated tissue. A Sinusoidal Segment (SS) does not map directly to a portion of a single sinusoid surrounded by hepatic endothelial cells, hepatocytes, etc. However, events occurring within a SS are strongly analogous to referent events within portions of sinusoids and adjacent tissue [15]. A vHPC maps to a conflation of relevant hepatocyte functionality that a small fraction of Dose encounters at a corresponding relative PV-to-CV location. A vLiver is a specified number of Monte Carlo (MC)-sampled vLobule variants.

vCompounds entering PV get exposed to a decreasing number of vHPCs as they approach the CV. A vLobule (Fig. 1A) is a directed acyclic graph with an SS agent at each node. The graph nodes are organized into five Layers. The number of nodes in Layers 1-5 are 45, 25, 20, 14, and 9, respectively. That structure maps directly to the polyhedral nature of hepatic lobules [16]. An SS (Fig. 1B) comprises a Core, Blood-Cell Interface, Endothelial Cell Space, Space of Disse, Hepatocyte Space, and Bile Space (not used during this study). Cell objects are software agents which occupy most of Endothelial Cell (99\%) and all of Hepatocyte Space.

A vCompound can have a specified chemical real-world counterpart, e.g., acetaminophen [10]. A vCompound maps to a small fraction of a referent oral drug dose. For this study, the Dose of vCompound is 50,000 objects. All Cells control vCompound entry and exit and contain a probability-specified number of Binders. Binders within vHPCs can be parameterized to Metabolize a Bound vCompound, but that is not done in this work. However, with probability $=0.1$, both vCompounds can be Bound (simulating a degree of non-specific binding). A Bound vCompound is released after 10 simulation cycles. During each simulation cycle, a fraction of vCompound in Mouse Body is transferred to PV. From PV, vCompounds enter Core and Interface space at the upstream end of all Layer-1 SSes and then percolate stochastically toward Hepatocyte Space and the CV [11,12], influenced by local flow specifications. They exit the last $\mathrm{SS}$, enter the CV, and then get moved to Mouse Body. Intra-Lobular vCompound movement is parametercontrolled and can be vCompound-specific. The simulated blood flow rate in Core is specified by ssFlowRate. Outside the Core, extra-Cellular vCompound movement is a biased random walk. Forward and lateral movement is controlled by forwardBias and lateralBias, separately specifiable for Core/Interface and the outer spaces (Endothelial, Space of Disse, etc.). Quasi-autonomous components recognize vCompounds and adjust their responses appropriately during each simulation cycle. For example, a vHPC recognizes that an adjacent vCompound outside the Cell has the property membrane crossing $=$ true, and, with a specified probability, allows it to enter; a vHPC recognizes that an unbound vCompound within that has the property pExit $=0$ (or 0.5 ), and does not allow it to exit (or with probability $=0.5$, allows it to exit).

\subsection{Measurements}

Measurements of features are made analogous to wet-lab counterparts to facilitate comparisons when wet-lab data are available. Because each execution's detailed specifications are determined using MC sampling, and the unfolding of events is stochastic, there can be considerable variability among measures from multiple executions. We count the number of vCompounds in several locations: 1) within Mouse Body, 2) extra-Cellular within Endothelial Cell Space, Space of Disse, or Hepatocyte Space, 3) intra-Cellular within vHPCs or 4) is elsewhere. The vLiver Extraction Ratio each cycle is defined as $1-$ [vCompound count that exited]/[vCompound count that entered]. A vCompound's Entry and Exit are mediated by each vHPC, based on the properties of the vCompound's real or hypothetical referent. 


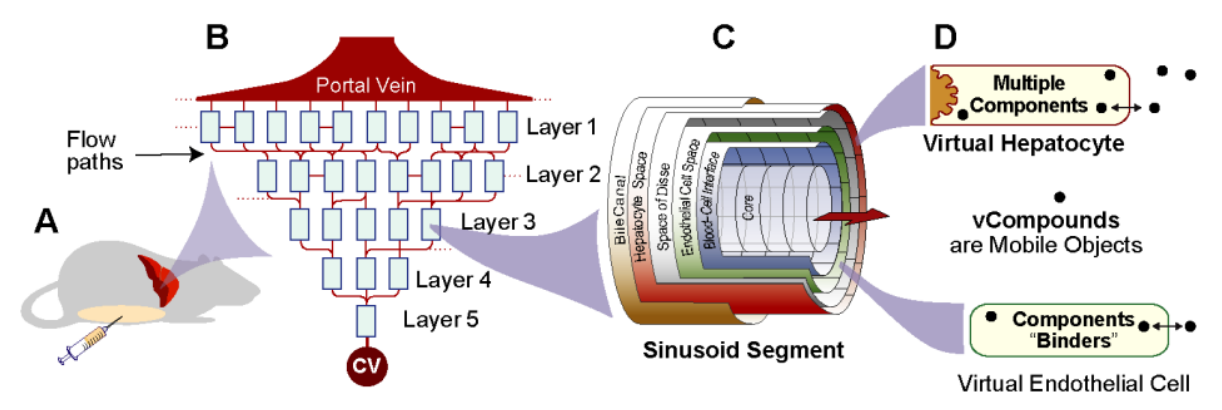

Figure 1. Virtual Mouse components. (A) A vMouse comprises a vLiver (a Monte Carlo-sampled number of vLobules), vBody, and a space to contain Dose. (B) Illustrated here is a portion of vLobule. Graph edges (red) designate flow connections within and between five Layers. (C) A multi-layered, quasi-3D SS maps to a portion of lobular tissue. During execution a SS functions as an analog of sinusoid components and features averaged across many actual lobules; SS dimensions are Monte Carlo (MC)-sampled to mimic lobular variability. An SS comprises a Core surrounded concentrically by five 2D grids. Mobile vCompound objects move within and between these grids. vCompounds enter and exit an SS via Core and Interface, percolate stochastically through accessible spaces influenced by configuration-controlled local flow parameters. vCompounds that exit to the CV are returned to Mouse Body. Measurements of vCompound in Mouse Body map to referent drug plasma levels. (D) Each vHPC and Endothelial Cell controls vCompound entry from and exit to adjacent spaces.

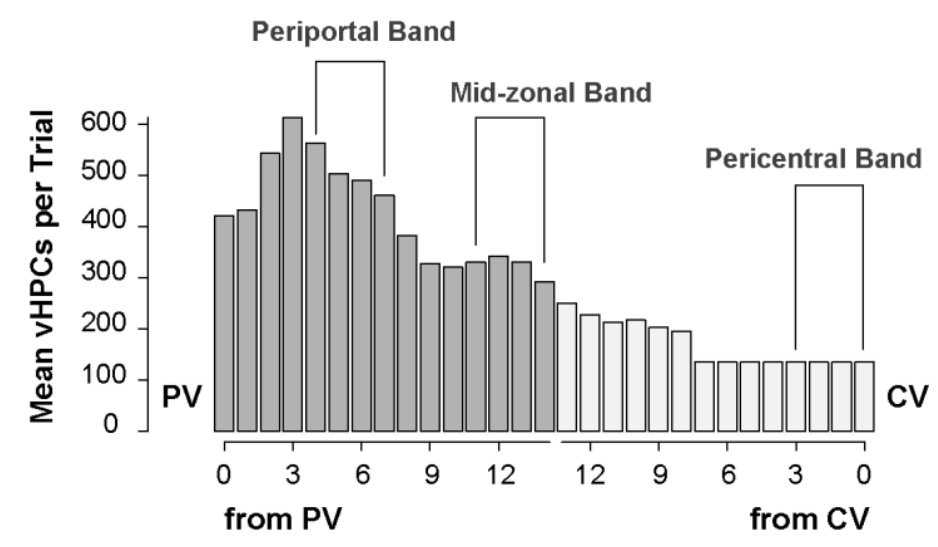

Figure 2. Mean number of vHPCs at each PV-to-CV location within an average vLobule. Dark gray are mean number of vHPCs at grid points $0-13$ (ordinal numbering) from the PV (designated dPV). Light gray are mean number of vHPCs at grid points 0-13 from the CV (designated dCV). Both dPV and dCV measures are needed because the shortest and longest PV-to-CV paths can be different for each execution. Together the two regions account for $100 \%$ of vHPCs. From left to right, measures are recorded within three bands: Periportal (dPV = 4-7), Mid-zonal ( $\mathrm{dPV}=11-14)$, and Pericentral $(\mathrm{dCV}=0-3)$. The mean vHPC counts at an average distance of $\mathrm{dPV}=0-3$ are smaller than the count at $\mathrm{dPV}=4$ because a few SSes in Layer 1 have MC sampled lengths of only 2-3 grid spaces. SS lengths in Layer 3 are fixed.

Results of Experiments help us understand how changes in pEnter/pExit impact vCompound dynamics within Sinusoidal Segments. We claim that the dynamics of vCompound-1 (pExit $=1$ with $\mathrm{pEnter}=0-1.0$ ) provides a plausible representation of the dynamics of real compounds exhibiting very small intrinsic clearance values; and that the dynamics of vCompound-2 (pExit $=0$ with $\mathrm{pEnter}=0$ 1.0) within vLobules provides a plausible representation of the dynamics of real compounds exhibiting very large intrinsic clearances values. Further, that changing pEnter maps directly to changing the unbound fraction of drug $\left(f_{u}\right)$ adjacent to HPCs. We define a vHPC entry event as one vHPC Exposure 
event. We conducted Experiments using pEnter $=0.0,0.1,0.3,0.5,0.8$ and 1.0. Although pExit for vCompound- $2=0$, it does not undergo Metabolism. We record each vHPC Exposure event for all vHPCs within a vLobule and within the three bands illustrated in Fig. 2.

\section{Results}

Various measures were recorded during virtual experiments using identical Doses of vCompound1 and -2. After about 55 Minutes vCompound-1 achieves a dynamic steady-state. Average amounts plateau in Mouse Body, vLiver, and within the three bands in Fig. 2. At dynamic steady-state, using pEnter $=0$, an average of $5.8 \%$ of the Dose ins within the vLiver in Extracellular spaces. For the conditions used, Increasing pEnter to $0.1,0.3,0.5,0.8$, and 1.0 , increases average steady-state amounts in vLivers to $6.6,6.9,7.3,8.9$, and $9.4 \%$ of Dose, respectively.

Measures of vCompound-1 average entry events per vHPC, per simulation cycle, and per MC trial (hereafter, simply Exposure Rates) are presented in Figs. 3 and 4. For pEnter $=1.0$, Exposure Rates within the Mid-zonal band (Fig. 3B) are 1.7-fold larger than in the Periportal band (Fig. 3A), whereas within the Pericentral band (Fig. 3C) Exposure Rates are 3.7-fold larger than within the Periportal band. When pEnter decreased to 0.8 (and 0.3), Mid-zonal Exposure Rates are 1.9-fold (2.2-fold) larger than Periportal Rates. Pericentral Exposure Rates are 4.2-fold (6.0-fold) larger than Periportal Rates. Those increases are a consequence of less vCompound-1 entering upstream vHPCs when pEnter is reduced from 1.0 to 0.8 (or to 0.3). Observing Exposure Rates increase from Periportal to Pericentral, which contradicts Hypothesis2 and is a direct consequence of the number of vHPCs decreasing from PV to CV (Fig. 2). Averaged over the six trials, there were 1931 vHPCs in the Periportal band (dPV = 4-7), 1271 in the Mid-zonal band $(\mathrm{dPV}=11-14)$, and 540 in the Pericentral band $(\mathrm{dCV}=0-3)$. The average amount of vCompound-1 within a vHPC was proportional to Exposure Rates. However, because pExit $=1.0$ the intra-vHPC amounts are small. Figure 4 show that dependence of average dynamic steady-state Exposure Rates on pEnter is nonlinear, and those the trends within each band are similar.
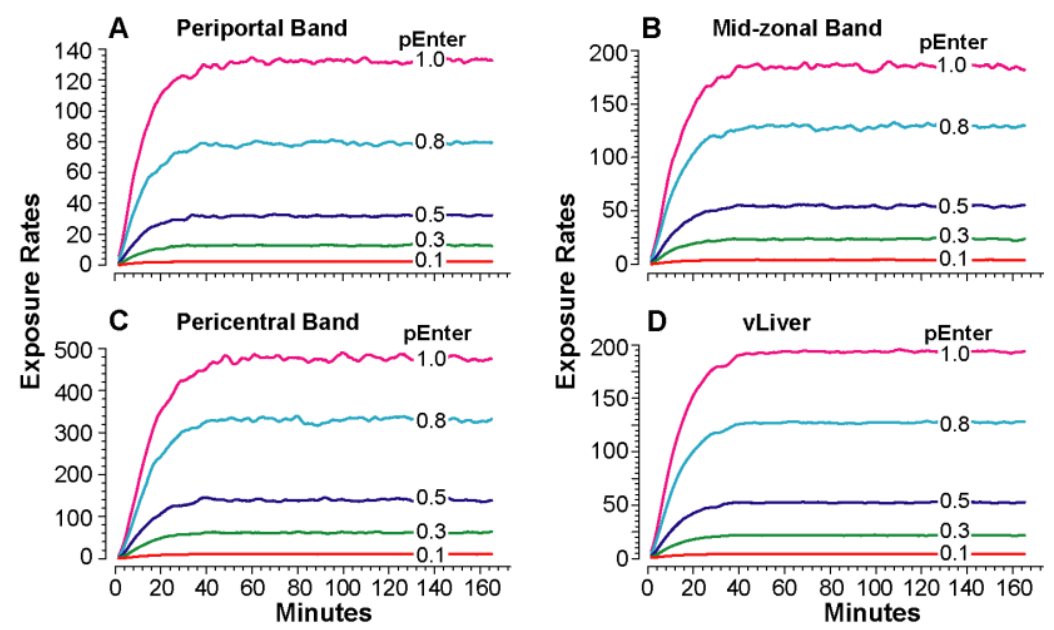

Figure 3. The influence of pEnter on Exposure Rates for vCompound-1. Measurements were recorded at the end of each simulation cycle and averaged over $6 \mathrm{MC}$ trials (A) within the Periportal band; (B) within the Midzonal band; (C) within the Pericentral band; and (D) for all vLiver locations. 

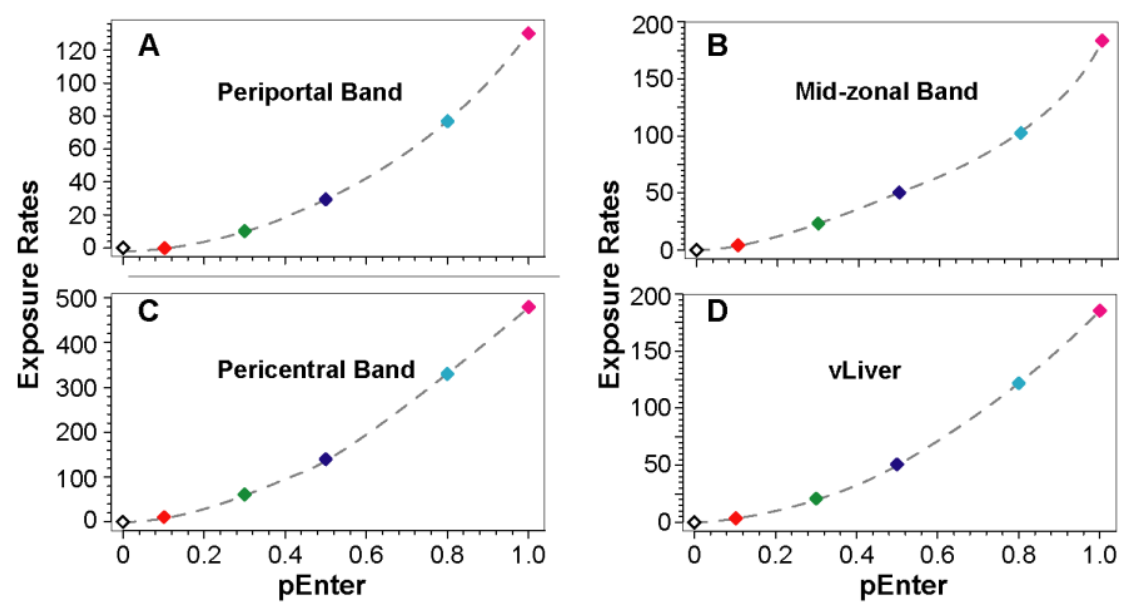

Figure 4. The dependence of average plateau Exposure Rates on pEnter is nonlinear. For each pEnter, mean plateau Exposure Rates from Fig. 3 were measured (A) within the Periportal band; (B) within the Mid-zonal band; (C) within the Pericentral band; and (D) for the average vLiver.

The results in Fig. 5 provide measures of vCompound-2 disposition. Within the variance, changing pEnter within the 0.3-1.0 range produces insignificant changes in pharmacokinetics in Mouse Body (Fig. 5A). Average measures of Extraction Ratio (Fig. 5B) plateau rapidly, and are somewhat robust to pEnter changes within the 0.3-1.0 range (Fig. 5B,C).
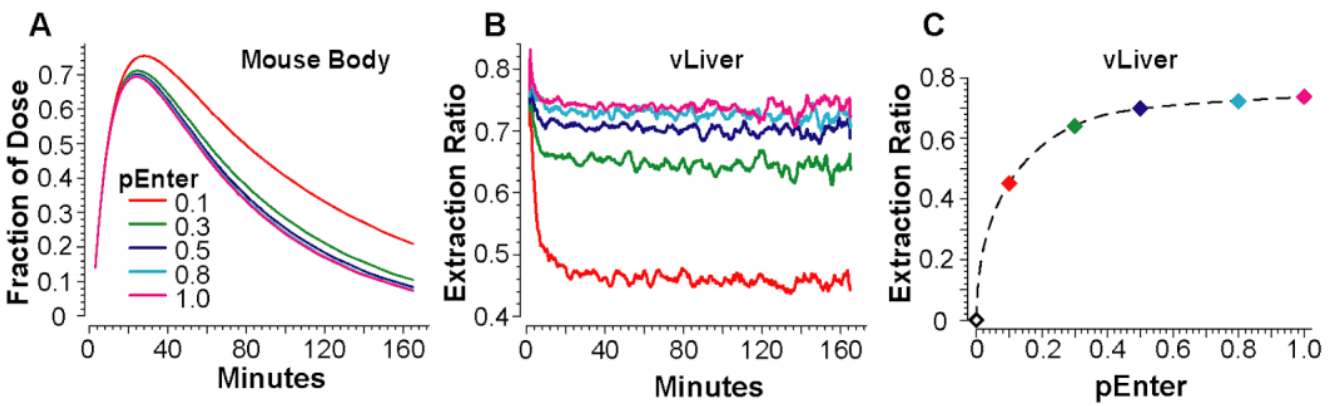

Figure 5. Features of vCompound-2 for each pEnter value. (A) Fraction of Dose in Mouse Body. (B) Centered moving average (window $=181$ cycles) for mean vLiver Extraction Ratio (6 MC trials). (C) Dependence of mean Extraction Ratio (120-160 minutes) from (B) on pEnter.

Because $\mathrm{pExit}=0$ for vCompound-2, a dynamic steady-state is not achieved (Fig. 6A-D). Because of vCompound-2 accumulation within vHPCs, measures per vHPC are dramatically larger than for vCompound-1. Having pExit $=0$ causes vCompound-2 to be trapped in upstream vHPCs, and that reduces the Exposure Rates to downstream vHPCs. That trapping dramatically changes vCompound-2 dynamics within and among SS spaces compared to vCompound-1. Consequenctly, for pEnter $=0.3$ to-1.0, the influence changing pEnter on Exposure Rates within the three bands is buffered. Average Exposure Rates for pEnter $=0.3-1.0$ are indistinguishable. Comparing those averages at 60 minutes, we see a 1.8-fold increase from Periportal to Pericentral bands, smaller than for vCompound-1. Note, 
however, that for the parallel tube model, Hypothesis-2 specifies that exposure rates decrease Periportal to Pericentral. For a comparable arrangement of vHPCs using pEnter $=1.0$, that decrease is $>80$ percent.

The amount of vCompound-2 accumulating within vHPCs within the three bands (Fig. 6E-H) is directly proportional to corresponding Exposure Rates. At 160 minutes, for $\mathrm{pEnter}=0.3-1.0$, the average intra-vHPC amount within the Mid-zonal band is 2.1 -fold larger than that within the Periportal band. At the same time, the average within the Pericentral band is 3.2-fold larger than that within the Periportal band. Hypothesis-2 specifies that the trend would be reversed, with Periportal > Pericentral. Accumulation differences are somewhat larger for $\mathrm{pExit}=0.1$ because less vCompound -2 is being trapped upstream; the average amount within the Mid-zonal band is 2.8 -fold larger than that within the Periportal band, whereas within the Pericentral band it is 5.5-fold larger than that within the Periportal band.
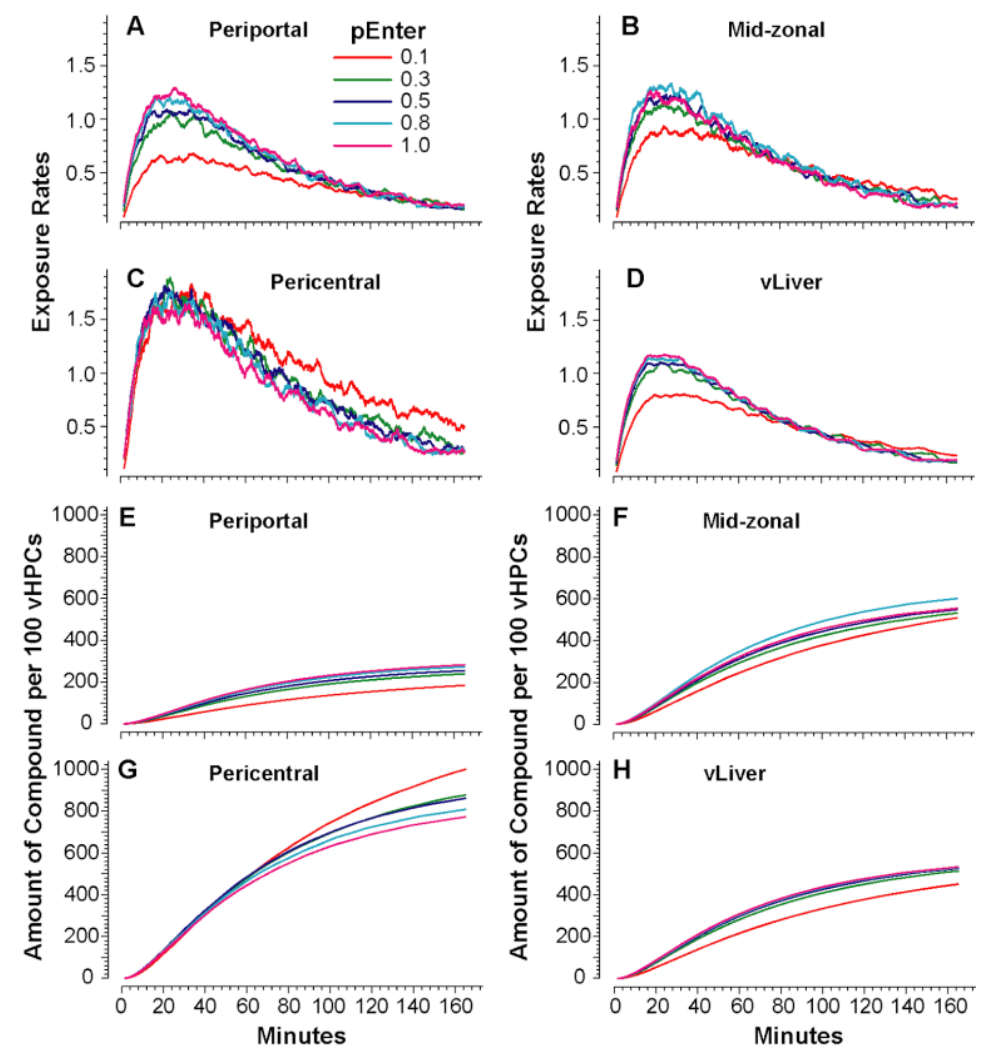

Figure 6. Average Exposure Rates and vHPC accumulation of vCompound-2. Measurements of average Exposure Rates (6 MC trials) for vCompound-2 for each pEnter value were recorded at the end of each simulation cycle (A) within the Periportal band; (B) within the Mid-zonal band; (C) within the Pericentral band; and (D) for the average vLiver. Measurements of average Amount of vCompound-2 were also recorded (E) within the Periportal band; (F) within the Mid-zonal band; (G) within the Pericentral band; and (H) for the average vLiver.

\section{Discussion}

Consequences of a valid Hypothesis-1 (the intralobular micro-anatomical organization of HPCs within hepatic lobules can be extrapolated away) include that 1) in vivo HPC exposure rates are essentially uniform, 2) exposure rates are directly proportional to $f_{u}$, the unbound fraction of drug in extra-HPC fluid, and 3) in vitro and in vivo HPC exposure rates are essentially the same. We reasoned that the disposition behavior within vLobules of vCompound-1 would be most supportive of the first 
and second consequence. This work did not challenge the third consequence. Neither consequence (1) nor (2) is supported by our results. Average Exposure Rates within the Pericentral band are greater than those in the Periportal band (Fig. 3). Further, the relationship between Exposure Rates and pEnter is nonlinear (Fig. 4). Given that evidence, we suggest that IVIVE inaccuracies may be due in part to using methods that are based on Hypothesis-1.

Hypothesis-2 applies when the parallel tube liver model is used to predict in vivo clearance. It is that drug concentration within the liver decreases exponentially PV-to-CV, and that at each PV-to-CV location, exposure rates are directly proportional to the unbound fraction of drug in extra-HPC fluid. As a consequence, one expects extraction ratio to be directly proportional to $f_{\mathrm{u}}$. We reasoned that the disposition behaviour within vLobules of vCompound-2 (highly permeable with maximum intrinsic clearance) would be most supportive of Hypothesis-2. However, none of those three attributes is supported by our results. At 160 minutes and for pEnter $=0.3-1.0$, the average intra-vHPC amount within the Pericentral band is larger than that within the Periportal band (Fig. 6). The dependence of Extraction ratio (Fig. 5) and location-dependent Exposure rates (Fig. 6) on pEnter are nonlinear and somewhat robust to pEnter changes within the 0.3-1.0 range. Given that evidence, we suggest that IVIVE methods employing the parallel tube liver model will result in underprediction of hepatic clearance. That evidence may help explain the frequently reported underprediction of in vivo hepatic clearance values when using established IVIVE methods.

The robustness of Exposure Rates, Extraction Ratio, and accumulation of vCompound-2 within vHPCs to pEnter changes within the 0.3-1.0 range (Figs. 5 and 6) merit explanation. That robustness is an emergent consequence of the concurrent mixed influences of vLiver structure (Fig. 1B), SS organization (Fig. 1C), and the complexity of vCompound-2 dynamics. The following illustrative example helps explain that robustness. Note that each of the three bands in Fig. 2 span four distance intervals containing vHPCs. Consider a vCompound- 2 having pEnter $=1$ that is adjacent to a vHPC at the upstream edge of one of the three bands. When given the opportunity to enter, the vHPC will allow it to do so and it becomes trapped. That is one vHPC Exposure event for that band. Consider a parallel experiment in which pEnter $=0.3$. A vCompound -2 is adjacent to a vHPC at the corresponding location within that same band. When given the opportunity to enter, it may fail to do so. During the next cycle, if it remains in the Space of Disse, it may have another opportunity to enter an adjacent or downstream vHPC. Again, it may fail to do so. Suppose that during the next cycle within that same band, the vCompound-2 is given a third opportunity to enter a vHPC, and it does so. That event counts as one exposure event for that band. Thus, in both cases, over the same interval and within the same band, there was one vCompound-2 Exposure event. However, for pEnter $=0.1$, the odds of entering a vHPC within that same band, within three simulation cycles, are significantly smaller (compared to pEnter $=$ $0.3)$.

Given the results presented, we suggest that IVIVE methods for predicting in vivo hepatic clearance can be improved by utilizing a liver model, such as the vLiver, that couples a biomimetic representation of the structural organization of HPCs along with biomimetic representations of intrahepatic vCompound dynamics.

\section{References}

[1] Choi GW, Lee YB, Cho HY. Interpretation of Non-Clinical Data for Prediction of Human Pharmacokinetic Parameters: In Vitro-In Vivo Extrapolation and Allometric Scaling. Pharmaceutics. 2019;11:E168.

[2] Bowman CM and Benet LZ. Hepatic clearance predictions from in vitro-in vivo extrapolation and BDDCS. Drug Metab Dispos. 2016;44:1731-35 
[3] Pelkonen O, and Turpeinen M. In vitro-in vivo extrapolation of hepatic clearance: biological tools, scaling factors, model assumptions and correct concentrations. Xenobiotica. 2007;37:1066-89.

[4] Hallifax D, Foster JA, and Houston JB. Prediction of human metabolic clearance from in vitro systems: retrospective analysis and prospective view. Pharm Res. 2010;27:2150-61.

[5] Wood FL, Houston JB, and Hallifax D. Clearance prediction methodology needs fundamental improvement: trends common to rat and human hepatocytes/microsomes and implications for experimental methodology. Drug Metab Dispos. 2017;45:1178-88.

[6] Tsamandouras N, Rostami-Hodjegan A, and Aarons L. Combining the 'bottom up' and 'top down' approaches in pharmacokinetic modelling: fitting PBPK models to observed clinical data. Br J Clin Pharmacol. 2015;79:48-55.

[7] Wood FL, Houston JB, and Hallifax D. Importance of the unstirred water layer and hepatocyte membrane integrity in vitro for quantification of intrinsic metabolic clearance. Drug Metab Dispos. 2018;46:268-78.

[8] Ye M, Nagar S, and Korzekwa K. A Physiologically Based pharmacokinetic model to predict the pharmacokinetics of highly protein-bound drugs and impact of errors in plasma protein binding. Biopharm Drug Dispos. 2016;37:123-41.

[9] Hunt C, Erdemir A, Lytton W, Mac Gabhann F, Sander E, Transtrum M, and Mulugeta L. The spectrum of Mechanism-Oriented models and methods for explanations of biological phenomena. Processes. 2018; 6:56.

[10] Smith AK, Petersen BK, Ropella GE, Kennedy RC, Kaplowitz N, Ookhtens M, and Hunt CA. Competing mechanistic hypotheses of acetaminophen-induced hepatotoxicity challenged by virtual experiments. PLoS Comput Biol. 2016;12:e1005253.

[11] Yan L, Ropella GE, Park S, Roberts MS, and Hunt CA. Modeling and simulation of hepatic drug disposition using a physiologically based, multi-agent in silico liver. Pharm Res. 2008a;25:1023-36.

[12] Yan L, Sheihk-Bahaei S, Park S, Ropella GE, and Hunt CA. (2008b) Predictions of hepatic disposition properties using a mechanistically realistic, physiologically based model. Drug Metab Dispos. 36:759-768.

[13] Bowman CM, and Benet LZ. An examination of protein binding and protein-facilitated uptake relating to in vitro-in vivo extrapolation. Eur J Pharm Sci. 2018;123:502-14.

[14] Chu X, Korzekwa K, Elsby R, Fenner K, Galetin A, Lai Y, Matsson P, Moss A, Nagar S, et al. Intracellular drug concentrations and transporters: Measurement, modeling, and implications for the liver. Clin Pharmacol Ther. 2013;94:126-41.

[15] Hunt CA, Ropella GE, Lam TN, and Gewitz AD. Relational grounding facilitates development of scientifically useful multiscale models. Theo Biol Med Model. 2011;8:35-66.

[16] Teutsch HF, Schuerfeld D, and Groezinger E. Three-dimensional reconstruction of parenchymal units in the liver of the rat. Hepatology. 1999;29:494-505. 